\title{
Computing Jump Conditions for the Immersed Interface Method Using Triangular Meshes
}

\author{
Sheng $\mathrm{Xu}^{*}$, Glen D. Pearson Jr. \\ Department of Mathematics, Southern Methodist University, Dallas, TX 75275-0156, \\ USA
}

\begin{abstract}
The immersed interface method (IIM) can be employed to solve many interface problems on fixed Cartesian grids by incorporating necessary interfaceinduced Cartesian jump conditions into numerical schemes. In this paper, we present a method to compute the necessary Cartesian jump conditions from given principal jump conditions using triangular mesh representation of an interface. The triangular mesh representation is simpler and robuster than interface parametrization for a complex or non-smooth interface. We test our method by using the computed Cartesian jump conditions in the IIM to solve a Poisson equation subject to an interface with the shape of a sphere, cube, cylinder or cone. The results demonstrate the expected second-order accuracy of the solution in the infinity norm.
\end{abstract}

Keywords: the immersed interface method, triangular meshes, jump conditions, Poisson solvers, complex geometries, non-smooth interfaces, Cartesian grid methods

\section{Introduction}

The immersed interface method (IIM) was first proposed by LeVeque and $\mathrm{Li}[2]$ to solve elliptic equations with discontinuous coefficients and singular sources. Since then it has been developed to be a general methodology for solving various interface problems and irregular-domain problems [3].

\footnotetext{
*Corresponding author

Email address: sxu@smu.edu (Sheng Xu)

$U R L:$ http://faculty.smu.edu/sxu (Sheng Xu)
} 
The key idea of the IIM is to incorporate interface-induced jump conditions into discretization schemes to achieve desired solution accuracy and solving efficiency. To give an example on how jump conditions are incorporated into a numerical scheme, we consider the central finite difference approximation of a derivative of a discontinuous function. Let $g(s)$ be a function with jump discontinuities at $s=l$ and $s=r$ that fall within a three-node stencil as $s_{i-1}<l<s_{i} \leq r<s_{i+1}$, where $s_{i-1}, s_{i}$ and $s_{i+1}$ are the stencil nodes satisfying $s_{i+1}-s_{i}=s_{i}-s_{i-1}=h$. The central finite difference scheme for $g^{\prime \prime}\left(s_{i}\right)$ on this stencil reads $[5,6]$

$$
\begin{aligned}
& \frac{\mathrm{d}^{2} g\left(s_{i}\right)}{\mathrm{d} s^{2}}=\frac{g\left(s_{i+1}\right)-2 g\left(s_{i}\right)+g\left(s_{i-1}\right)}{h^{2}}+O\left(h^{2}\right) \\
& -\frac{1}{h^{2}}\left(\sum_{n=0}^{3} \frac{-\left[g^{(n)}(l)\right]}{n !}\left(s_{i-1}-l\right)^{n}+\sum_{n=0}^{3} \frac{\left[g^{(n)}(r)\right]}{n !}\left(s_{i+1}-r\right)^{n}\right),
\end{aligned}
$$

where $\left[g^{(n)}(l)\right]=g^{(n)}\left(l^{+}\right)-g^{(n)}\left(l^{-}\right)$denotes the jump conditions of the n-th derivatives of $g(s)$ at $s=l$, and similarly $\left[g^{(n)}(r)\right]$ the jump conditions at $s=r$. This example shows that we can modify a standard finite difference scheme to approximate derivatives of a discontinuous function by adding some extra terms that involve necessary jump conditions. In particular, a PDE for an interface problem can be discretized on a fixed Cartesian grid by incorporating into standard discretization schemes the jump conditions of the solution and its derivatives with respect to Cartesian coordinates. We name these jump conditions Cartesian jump conditions.

For the function $p$, we call

$$
[p], \quad\left[\frac{\partial p}{\partial \mathbf{n}}\right], \quad[\Delta p]
$$

the principal jump conditions, which are the jump conditions of $p$, the normal derivative of $p$ and the Laplacian of $p$ across an interface, respectively, where $\mathbf{n}$ denotes a unit normal vector for the interface, and a jump condition is hereafter defined as $[\cdot]=(\cdot)_{\mathbf{n}^{+}}-(\cdot)_{\mathbf{n}^{-}}$with $\mathbf{n}^{+}$and $\mathbf{n}^{-}$denoting right and left limits respectively. These principal jump conditions can often be derived from the physics of a problem. The goal of this paper is to obtain from the given principal jump conditions the following Cartesian jump conditions

$$
[p], \quad\left[\frac{\partial p}{\partial x_{i}}\right], \quad\left[\frac{\partial^{2} p}{\partial x_{i} \partial x_{j}}\right]
$$


where $x_{i}$ and $x_{j}(i, j \in\{1,2,3\})$ are Cartesian coordinates in 3D.

In [6], a systematic approach based on interface parametrization was proposed to derive the Cartesian jump conditions from the principal jump conditions. However, interface parametrization is practically difficult for complex or non-smooth interfaces. In this paper, we propose a method to compute the Cartesian jump conditions using triangular mesh representation of an interface, which is more flexible and robust to deal with complex or non-smooth interfaces.

To test our method, we use the Cartesian jump conditions computed by the method in the IIM to solve the Poisson equation $\Delta p=f$ subject to the given principal jump conditions across a closed interface in 3D. Based on Eqn. (1), the Poisson equation can be discretized on a uniform Cartesian grid as

$$
\Delta_{h} p_{i j k}=f_{i j k}+C_{i j k}
$$

where $\Delta_{h}$ is the standard seven-point discrete Laplacian at the grid point $(i, j, k), h$ is the spatial step of the grid, and $C_{i j k}$ denotes the extra terms involving the necessary Cartesian jump conditions. The jump contribution $C_{i j k}$ is nonzero only if the interface cuts through the seven-point Laplacian stencil at the grid point $(i, j, k)$.

According to Eqn. (1), the local truncation error of the seven-point discrete Laplacian is $O(h)$ if the jump conditions of the third-order Cartesian derivatives are not included. However, it is difficult or costly to obtain the jump conditions of the third-order or higher Cartesian derivatives. Fortunately, second-order accuracy of the solution in the infinity norm can still be achieved in solving a Poisson equation subject to an interface if the local truncation error is reduced from $O\left(h^{2}\right)$ to $O(h)$ only for stencils cut by the interface [1]. So we only need to compute the Cartesian jump conditions listed above. Realizing that $\left(s_{i-1}-l\right)$ and $\left(s_{i-1}-r\right)$ in Eqn. (1) is at most $O(h)$, we need to compute $\left[\frac{\partial p}{\partial x_{i}}\right]$ with $O\left(h^{2}\right)$ accuracy and $\left[\frac{\partial^{2} p}{\partial x_{i} \partial x_{j}}\right]$ with $O(h)$ accuracy.

One motivation of the work in this paper is to solve a fluid flow around moving rigid objects on a fixed Cartesian grid using the IIM with boundary condition capturing $[8,10]$. The flow is formulated such that the rigid objects are replaced by the virtual fluid enclosed by singular forces concentrating on the surfaces of the objects via the Dirac delta function, which induce jump discontinuities in the velocity and the pressure across the interfaces between 
the virtual and real fluids. The principal jump conditions for the velocity and the pressure are related with the singular forces [6], which can be determined explicitly in the boundary condition capturing approach $[8,9,10]$. Using the method proposed in this paper, we can obtain the Cartesian jump conditions explicitly. They appear at the right-hand sides of linear systems resulting from the discretization of the Navier-Stokes equations without modifying the coefficient matrices corresponding to the background standard numerical schemes, so standard fast solvers such as FFT solvers can be employed to solve the linear systems. Linnick and Fasel [4] presented a high-order IIM in which Cartesian jump conditions are directly computed from unknown solutions using one-sided finite difference schemes. Since their Cartesian jump conditions are solution-dependent, the coefficient matrices of their linear systems are modified and iterative solvers are required.

\section{Cartesian Jump Conditions}

Suppose that a triangular mesh representation of a closed interface is given. See Fig. 1 for examples. Suppose that the edge lengths of the triangular mesh is of $O(h)$. Suppose that the principal jump conditions $[p],\left[\frac{\partial p}{\partial \mathbf{n}}\right]$, and $[\Delta p]$ are given at appropriate locations (specified later) on the triangular mesh. The Cartesian jump condition $[p]$ is given as a principal jump condition. We compute the Cartesian jump conditions $\left[\frac{\partial p}{\partial x_{i}}\right]$ and $\left[\frac{\partial^{2} p}{\partial x_{i} \partial x_{j}}\right]$ at each vertex of the triangular mesh.

According to Eqn. (1), we need to compute $\left[\frac{\partial p}{\partial x_{i}}\right]$ with at least $O\left(h^{2}\right)$ accuracy and $\left[\frac{\partial^{2} p}{\partial x_{i} \partial x_{j}}\right]$ with at least $O(h)$ accuracy such that the local truncation error of the seven-point discrete Laplacian is at most $O(h)$ for stencils cut by the interface, as already stated in Section 1 .

To facilitate later presentation, we consider an arbitrarily chosen triangular panel $\triangle A B C$ in Fig. 2 (a) and an arbitrarily chosen vertex $A$ in Fig. 2 (b).

\section{1. $O\left(h^{2}\right)$ computation of $\left[\frac{\partial p}{\partial x_{i}}\right]$}

For an arbitrary triangular panel $\triangle A B C$ shown in Fig. 2 (a), we first introduce some notations in the computation of $\left[\frac{\partial p}{\partial x_{i}}\right]$. We denote the middle points of the edges $A B, B C$ and $C A$ as $D, E$ and $F$, respectively, and the intersection of $A E$ and $D F$ as $M$ (which is the middle point of $A E$ and $D F$ ). 

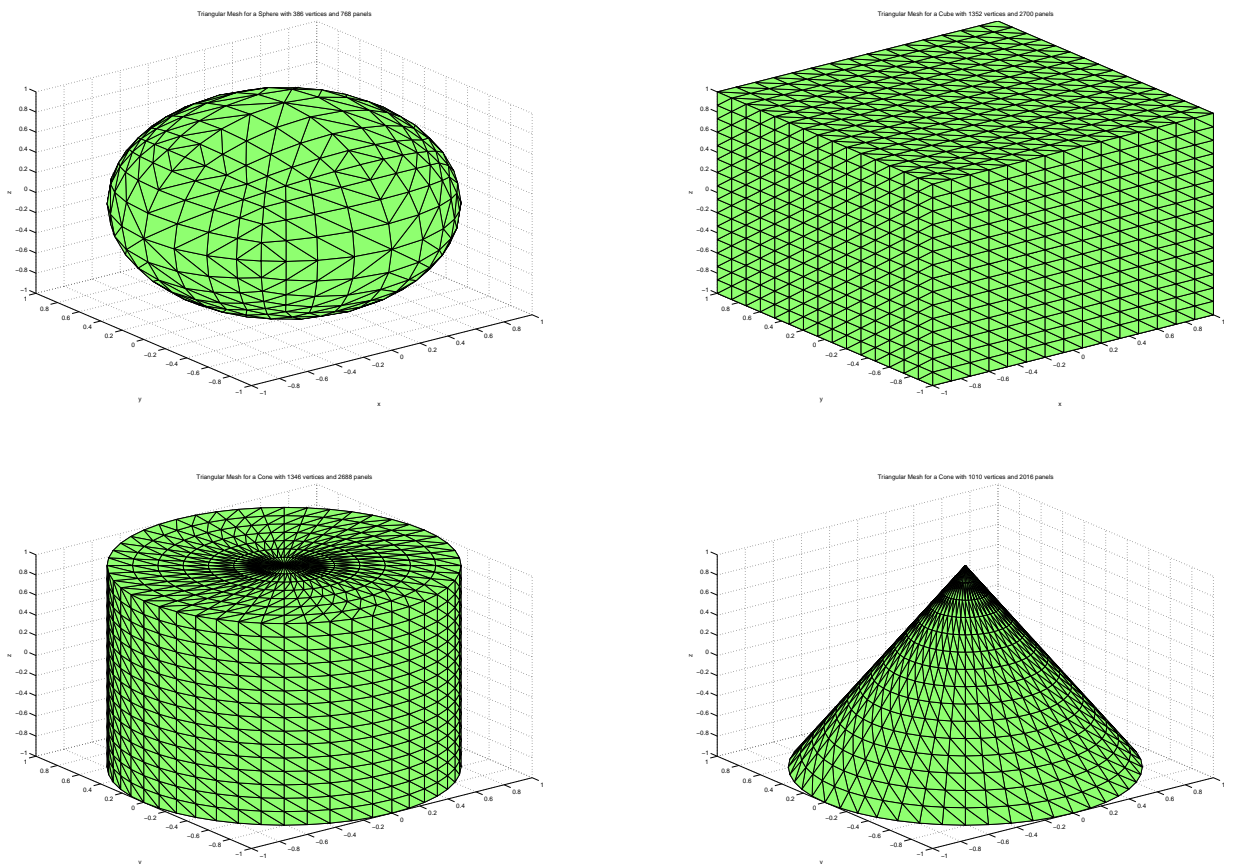

Figure 1: Triangular meshes for a sphere, a cube, a cylinder and a cone.

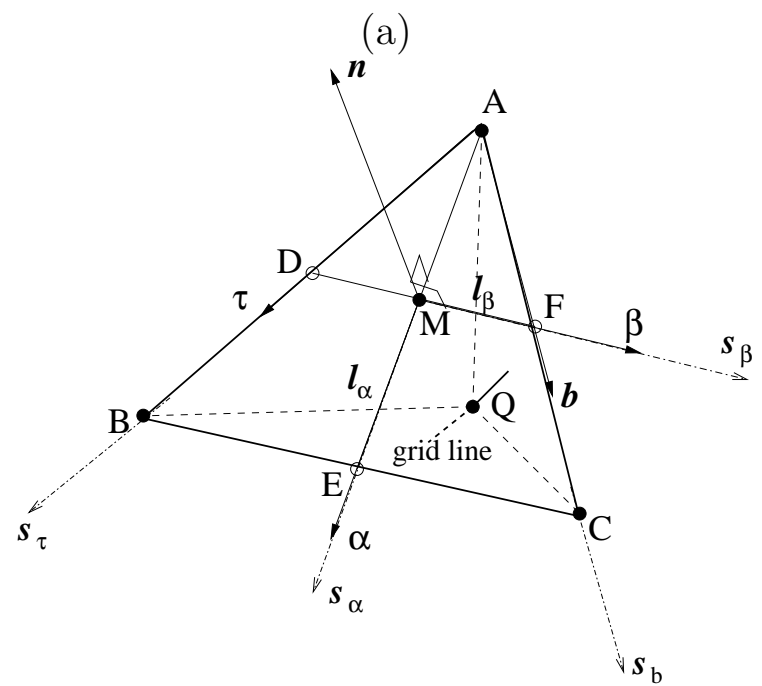

(b)

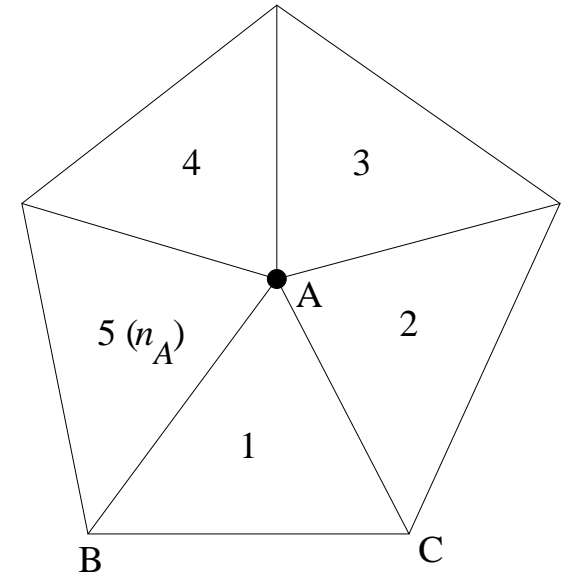

Figure 2: (a) A triangular panel $\triangle A B C$ and various points, lines and vectors associated with it. (b) A vertex $A$ shared by $n_{A}=5$ surrounding triangular panels. 
We let $\ell_{\alpha}=|M E|, \ell_{\beta}=|M F|\left(\ell_{\alpha}\right.$ and $\ell_{\beta}$ are of $\left.O(h)\right), s_{\alpha}$ and $s_{\beta}$ be the axes along $M E$ and $M F$ with unit vectors $\boldsymbol{\alpha}$ and $\boldsymbol{\beta}$, respectively, and $\mathbf{n}$ be the unit normal vector of the panel $\triangle A B C$ pointing outside of the closed interface. We suppose that the vertices $A, B$ and $C$ are ordered such that $\mathbf{n}$ is in the direction of $\boldsymbol{\alpha} \times \boldsymbol{\beta}$.

Assume that we know the principal jump conditions $\left[\frac{\partial p}{\partial \mathbf{n}}\right]$ at the point $M$ with at least $O\left(h^{2}\right)$ accuracy and the principal jump conditions $[p]$ at the points $A, B, C, D, E$ and $F$ with at least $O\left(h^{3}\right)$ accuracy. Our first step is to compute $\left[\frac{\partial p}{\partial x_{i}}\right]$ at the point $M$ with $O\left(h^{2}\right)$ accuracy. We have

$$
\begin{aligned}
\boldsymbol{\alpha} \cdot[\nabla p]_{M} & =\left[\frac{\partial p}{\partial s_{\alpha}}\right]_{M}=\frac{[p]_{E}-[p]_{A}}{2 \ell_{\alpha}}+O\left(h^{2}\right), \\
\boldsymbol{\beta} \cdot[\nabla p]_{M} & =\left[\frac{\partial p}{\partial s_{\beta}}\right]_{M}=\frac{[p]_{F}-[p]_{D}}{2 \ell_{\beta}}+O\left(h^{2}\right), \\
\mathbf{n} \cdot[\nabla p]_{M} & =\left[\frac{\partial p}{\partial \mathbf{n}}\right]_{M} .
\end{aligned}
$$

Eqs. $(2,3,4)$ form the following linear system

$$
\left(\begin{array}{ccc}
\alpha_{1} & \alpha_{2} & \alpha_{3} \\
\beta_{1} & \beta_{2} & \beta_{3} \\
n_{1} & n_{2} & n_{3}
\end{array}\right)\left(\begin{array}{c}
{\left[\frac{\partial p}{\partial x_{1}}\right]} \\
{\left[\frac{\partial p}{\partial x_{2}}\right]} \\
{\left[\frac{\partial p}{\partial x_{3}}\right]}
\end{array}\right)=\left(\begin{array}{c}
\frac{[p]_{E}-[p]_{A}}{2 \ell_{\alpha}} \\
\frac{[p]_{F}-[p]_{D}}{2 \ell_{\beta}} \\
{\left[\frac{\partial p}{\partial \mathbf{n}}\right]}
\end{array}\right) .
$$

This linear system can be solved analytically to give $[\nabla p]$ at the point $M$ with $O\left(h^{2}\right)$ accuracy. We apply this process for all the panels on our mesh.

Next, we use $[\nabla p]$ at the interior point $M$ of each panel to compute $[\nabla p]$ at all the vertices. For the single panel shown in Fig.2 (a), linear interpolation gives

$$
2[\nabla p]_{A}+[\nabla p]_{B}+[\nabla p]_{C}=4[\nabla p]_{M}+O\left(h^{2}\right)
$$

So by repeating the linear interpolation for all the adjacent panels to the vertex $A$ and then doing summation of the results, we can form a linear 
equation

$$
2 n_{A}[\nabla p]_{A}+2[\nabla p]_{B}+2[\nabla p]_{C}+\cdots=4 \sum_{i=1}^{n_{A}}[\nabla p]_{M_{i}}
$$

where $n_{A}$ is the number of the adjacent vertices (or panels) to the vertex $A$, as demonstrated in Fig.2 (b), and the factor 2 in front of $[\nabla p]_{B}$ and $[\nabla p]_{C}$ comes from the fact that the edge $B C$ is shared by two neighboring panels. Assembling such linear equations for all the vertices forms the linear system

$$
\left(\begin{array}{cccc}
n_{A} & 1 & 1 & \cdots \\
1 & n_{B} & 1 & \cdots \\
1 & 1 & n_{C} & \cdots \\
& & \vdots &
\end{array}\right)\left(\begin{array}{c}
{[\nabla p]_{A}} \\
{[\nabla p]_{B}} \\
{[\nabla p]_{C}} \\
\vdots
\end{array}\right)=2\left(\begin{array}{c}
\sum_{i=1}^{n_{A}}[\nabla p]_{M_{i}} \\
\sum_{i=1}^{n_{B}}[\nabla p]_{M_{i}} \\
\sum_{i=1}^{n_{C}}[\nabla p]_{M_{i}} \\
\vdots
\end{array}\right)
$$

which can be solved using the conjugate gradient (CG) method as its coefficient matrix is symmetric positive definite (SPD). The solution is $[\nabla p]$ at all the vertices with $O\left(h^{2}\right)$ accuracy. This linear system is not coupled with $p$ if the given principal jump conditions are explicitly known and not coupled with $p$, which is the case in the IIM with boundary condition capturing for fluid-solid interaction $[8,10]$.

Denote the coefficient matrix as $G$. To compute the matrix-vector product $G q=d$ for a known approximation $q$ of the solution in the CG method, we do not need to construct or store $G$ or find surrounding panels for each vertex. Write down $G q=d$ as

$$
\left(\begin{array}{cccc}
n_{A} & 1 & 1 & \ldots \\
1 & n_{B} & 1 & \ldots \\
1 & 1 & n_{C} & \ldots \\
& & \vdots &
\end{array}\right)\left(\begin{array}{c}
q_{A} \\
q_{B} \\
q_{C} \\
\vdots
\end{array}\right)=\left(\begin{array}{c}
d_{A} \\
d_{B} \\
d_{C} \\
\vdots
\end{array}\right)
$$

We can easily compute the matrix vector product $d$ using the following algorithm 


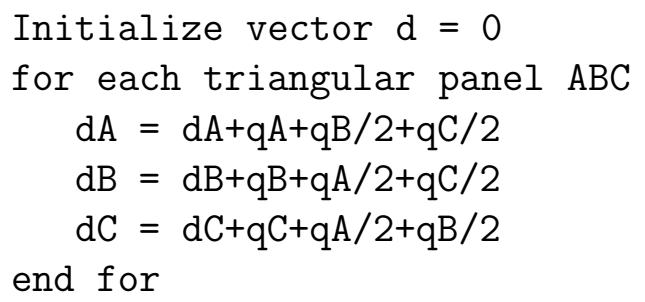

The division by 2 above comes from the fact that any two adjacent panels share a common edge.

\section{2. $O(h)$ computation of $\left[\frac{\partial^{2} p}{\partial x_{i} \partial x_{j}}\right]$}

To compute $\left[\frac{\partial^{2} p}{\partial x_{i} \partial x_{j}}\right]$ with $O(h)$ accuracy, we use $\left[\frac{\partial p}{\partial x_{i}}\right]$ computed at each vertex with $O\left(h^{2}\right)$ accuracy in Section 2.1 and the principal jump condition $[\Delta p]$ supposed to be known at each vertex with at least $O(h)$ accuracy.

For an arbitrary triangular panel $\triangle A B C$ illustrated in Fig. 2 (a), below we present how to compute $\left[\frac{\partial^{2} p}{\partial x_{i} \partial x_{j}}\right]$ with $O(h)$ accuracy at an arbitrary vertex of the panel, say the vertex $A$. In Fig. $2(\mathrm{a}), s_{\tau}$ and $s_{b}$ are the axes along $A B$ and $A C$ with unit vectors $\tau$ and $\mathbf{b}$. We approximate the following derivatives along $s_{\tau}$ and $s_{b}$ at the vertex $A$ using one-sided finite difference approximations

$$
\begin{aligned}
& \frac{\partial[\nabla p]}{\partial s_{\tau}}=\tau_{1} \frac{\partial[\nabla p]}{\partial x_{1}}+\tau_{2} \frac{\partial[\nabla p]}{\partial x_{2}}+\tau_{3} \frac{\partial[\nabla p]}{\partial x_{3}}=\frac{[\nabla p]_{B}-[\nabla p]_{A}}{|A B|}+O(h),(10) \\
& \frac{\partial[\nabla p]}{\partial s_{b}}=b_{1} \frac{\partial[\nabla p]}{\partial x_{1}}+b_{2} \frac{\partial[\nabla p]}{\partial x_{2}}+b_{3} \frac{\partial[\nabla p]}{\partial x_{3}}=\frac{[\nabla p]_{C}-[\nabla p]_{A}}{|A C|}+O(h) .(11)
\end{aligned}
$$

Combining Eqs. $(10,11)$ with the following given principal jump condition at the vertex $A$

$$
\left[\frac{\partial^{2} p}{\partial x_{1}^{2}}\right]+\left[\frac{\partial^{2} p}{\partial x_{2}^{2}}\right]+\left[\frac{\partial^{2} p}{\partial x_{3}^{2}}\right]=[\Delta p]
$$

we obtain the following over-determined linear system for $\left[\frac{\partial^{2} p}{\partial x_{i} \partial x_{j}}\right]$ at the ver- 
$\operatorname{tex} A$

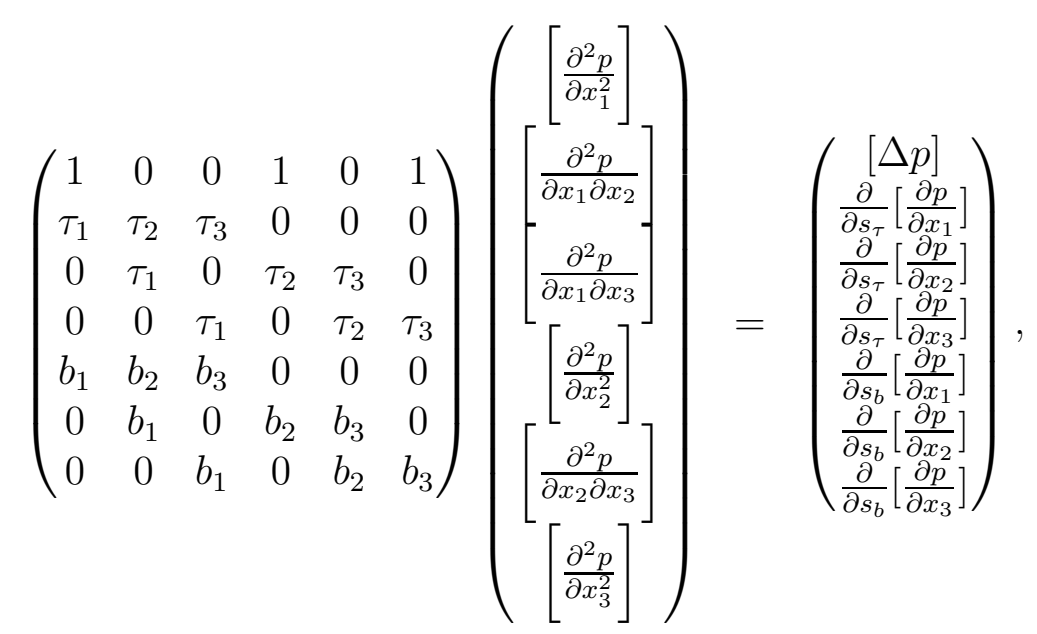

which has a unique solution and can be solved analytically by Gaussian elimination $[6,7]$.

Using this process, We can solve $\left[\frac{\partial^{2} p}{\partial x_{i} \partial x_{j}}\right]$ at the vertex $A$ from all the $n_{A}$ panels surrounding the vertex $A$ as shown in Fig. 2 (b). We use a weighted average to obtain the final $\left[\frac{\partial^{2} p}{\partial x_{i} \partial x_{j}}\right]$ at the vertex $A$ as the following

$$
\left[\frac{\partial^{2} p}{\partial x_{i} \partial x_{j}}\right]=\frac{\sum_{k=1}^{n_{A}}\left(w_{k}\left[\frac{\partial^{2} p}{\partial x_{i} \partial x_{j}}\right]_{k}\right)}{\sum_{k=1}^{n_{A}} w_{k}}
$$

where $w_{k}$ is a weight and $\left[\frac{\partial^{2} p}{\partial x_{i} \partial x_{j}}\right]_{k}$ denotes $\left[\frac{\partial^{2} p}{\partial x_{i} \partial x_{j}}\right]$ computed from the panel $k$ surrounding the vertex $A$. The weight $w_{k}$ is chosen to be $w_{k}=A_{k}^{-1}$, where $A_{k}$ is the area of the panel $k$, such that a smaller panel has a larger weight. Since the vertices of a smaller panel are closer in general, the weighted average improves the accuracy in computing $\left[\frac{\partial^{2} p}{\partial x_{i} \partial x_{j}}\right]$.

\section{Interpolation at Panel-Grid Intersections}

We now present how to use the computed Cartesian jump conditions in the IIM to solve an interface problem in 3D. An interface is represented by a triangular mesh with mesh size of order $O(h)$. 
We need to distinguish grid points inside and outside the interface. This can be done by the ray casting scheme. As indicated in Section 1, we need to identify finite difference stencils which are cut by the interface. This can be done by finding all the intersections (without omission or repetition) between Cartesian grid lines and all the triangular panels of the triangular mesh [7]. In order to determine the jump contributions to those finite difference stencils using Eqn. (1), we need to know the necessary Cartesian jump conditions at all these intersections. Below we present how to interpolate a Cartesian jump condition at an intersections from the surrounding vertices with required accuracy.

Let the point $Q$ in Fig. 2 (a) be an intersection between a Cartesian grid line and the triangular panel $\triangle A B C$ of the interface. We use $f$ to denote a Cartesian jump condition $\left[\frac{\partial p}{\partial x_{i}}\right]$ or $\left[\frac{\partial^{2} p}{\partial x_{i} \partial x_{j}}\right]$. We denote the known values of $f$ at the vertices $A, B$ and $C$ of the panel as $f_{A}, f_{B}$ and $f_{C}$, respectively. We can interpolate the value of $f$ at $Q$, denoted as $f_{Q}$, using the following formula

$$
f_{Q}=\frac{\|\overrightarrow{Q B} \times \overrightarrow{Q C}\| f_{A}+\|\overrightarrow{A Q} \times \overrightarrow{A C}\| f_{B}+\|\overrightarrow{A B} \times \overrightarrow{A Q}\| f_{C}}{\|\overrightarrow{A B} \times \overrightarrow{A C}\|}+O\left(h^{2}\right)
$$

The interpolation is of second order accuracy, so it keeps the desired accuracy in $\left[\frac{\partial p}{\partial x_{i}}\right]$ or $\left[\frac{\partial^{2} p}{\partial x_{i} \partial x_{j}}\right]$.

We now show how to interpolate $[p]$ at the intersection $Q$, denoted as $[p]_{Q}$. According to Eqn. (1), we need $[p]_{Q}$ with $O\left(h^{3}\right)$ accuracy so that the local truncation error of the seven-point discrete Laplacian is $O(h)$. For an arbitrary triangular panel $\triangle A B C$ shown in Fig. 2 (a), suppose we are given the principal jump condition $[p]$ at the vertices $A, B$ and $C$ and the edge middle points $D, E$ and $F$ with at least $O\left(h^{3}\right)$ accuracy. We can interpolate $[p]_{Q}$ with $O\left(h^{3}\right)$ accuracy using the following third order quadratic interpolation scheme

$$
[p]=c_{0}+c_{1} \xi+c_{2} \eta+c_{3} \xi^{2}+c_{4} \eta^{2}+c_{5} \xi \eta+O\left(h^{3}\right),
$$

where $\xi=s_{\alpha} / \ell_{\alpha}$ and $\eta=s_{\beta} / \ell_{\beta}$ are nondimensional coordinates along the $s_{\alpha}$ and $s_{\beta}$ axes in Fig. $2(\mathrm{a})$, and $c^{\prime} s$ are coefficients. The points $A, B, C, D, E$ and $F$ are represented by the nondimensional coordinates as $A(-1,0), B(1,-2)$, $C(1,2), D(0,-1), E(1,0)$ and $F(0,1)$, and $[p]$ at these points are given as $[p]_{A},[p]_{B},[p]_{C},[p]_{D},[p]_{E}$ and $[p]_{F}$. So we have the following linear system to 
determine the coefficients $c^{\prime} s$

$$
\left(\begin{array}{cccccc}
1 & -1 & 0 & 1 & 0 & 0 \\
1 & 1 & -2 & 1 & 4 & -2 \\
1 & 1 & 2 & 1 & 4 & 2 \\
1 & 0 & -1 & 0 & 1 & 0 \\
1 & 1 & 0 & 1 & 0 & 0 \\
1 & 0 & 1 & 0 & 1 & 0
\end{array}\right)\left(\begin{array}{l}
c_{0} \\
c_{1} \\
c_{2} \\
c_{3} \\
c_{4} \\
c_{5}
\end{array}\right)=\left(\begin{array}{l}
{[p]_{A}} \\
{[p]_{B}} \\
{[p]_{C}} \\
{[p]_{D}} \\
{[p]_{E}} \\
{[p]_{F}}
\end{array}\right)
$$

which has the solution

$$
\begin{aligned}
& c_{0}=\frac{[p]_{D}+[p]_{F}}{2}-\frac{[p]_{B}+[p]_{C}-2[p]_{E}}{8}, \\
& c_{1}=\frac{[p]_{E}-[p]_{A}}{2}, \quad c_{2}=\frac{[p]_{F}-[p]_{D}}{2} \\
& c_{3}=\frac{[p]_{A}-[p]_{D}-[p]_{F}}{2}+\frac{[p]_{B}+[p]_{C}+2[p]_{E}}{8}, \\
& c_{4}=\frac{[p]_{B}+[p]_{C}-2[p]_{E}}{8}, \quad c_{5}=\frac{[p]_{C}-[p]_{B}}{4}-\frac{[P]_{F}-[p]_{D}}{2} .
\end{aligned}
$$

To use the interpolation scheme in Eqn. (16) to interpolate $[p]_{Q}$, we need to know the nondimensional coordinates $\left(\xi_{Q}, \eta_{Q}\right)$ of the point $Q$. From $\overrightarrow{M Q}=$ $\xi_{Q} \ell_{\alpha} \boldsymbol{\alpha}+\eta_{Q} \ell_{\beta} \boldsymbol{\beta}$, we obtain

$$
\xi_{Q}=\frac{(\overrightarrow{M Q} \times \boldsymbol{\beta}) \cdot \mathbf{n}}{\ell_{\alpha}(\boldsymbol{\alpha} \times \boldsymbol{\beta}) \cdot \mathbf{n}}, \quad \eta_{Q}=\frac{(\overrightarrow{M Q} \times \boldsymbol{\alpha}) \cdot \mathbf{n}}{\ell_{\beta}(\boldsymbol{\beta} \times \boldsymbol{\alpha}) \cdot \mathbf{n}} .
$$

\section{Numerical Tests}

To test our computation of the Cartesian jump conditions, we construct the function $p$ analytically such that it is discontinuous across a closed interface $\Gamma$ as

$$
p\left(x_{1}, x_{2}, x_{3}\right)= \begin{cases}\sin \left(x_{1}\right) \sin \left(x_{2}\right) \sin \left(x_{3}\right), & \text { outside } \Gamma, \\ \mathrm{e}^{-x_{1}} \mathrm{e}^{-x_{2}} \mathrm{e}^{-x_{3}}, & \text { inside } \Gamma .\end{cases}
$$

We represent the interface using a triangular mesh with mesh size of $O(h)$. We compute the Cartesian jump conditions from the analytical principal jump conditions using the processes presented in Sections 2.1 and 2.2. We then compare the computed results with the analytical Cartesian jump conditions. Fig. 3 shows that we obtain the desired second order accuracy for 
(a)

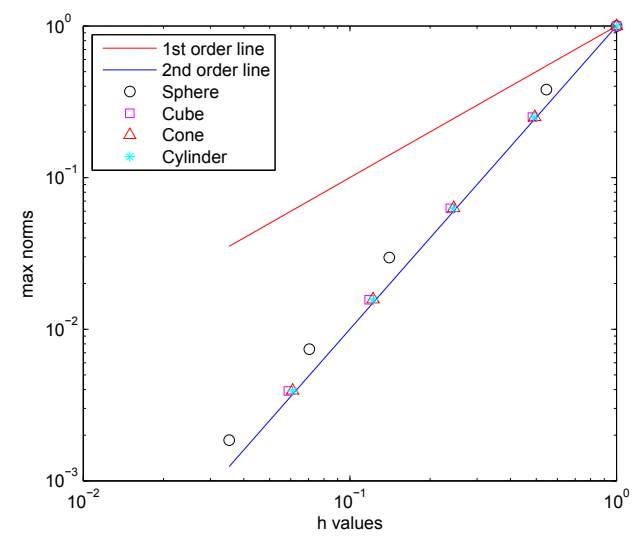

(b)

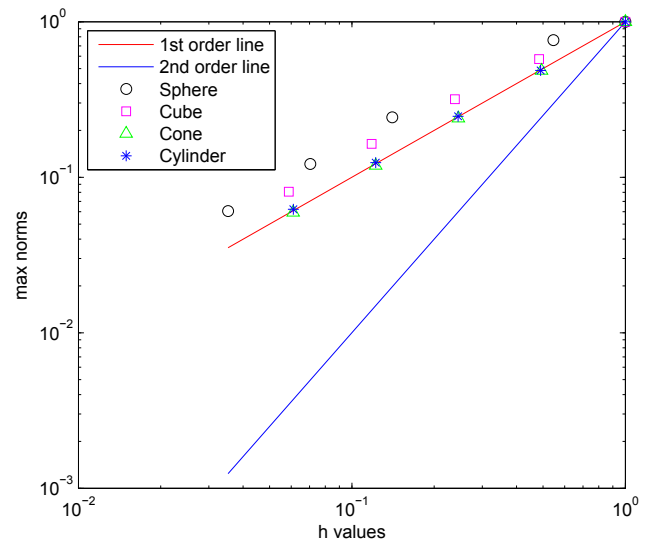

Figure 3: The scaled infinity norms of the errors versus the scaled mesh sizes for (a) $\left[\frac{\partial p}{\partial x_{1}}\right]$ and (b) $\left[\frac{\partial p^{2}}{\partial x_{1}^{2}}\right]$.

$\left[\frac{\partial p}{\partial x_{1}}\right]$ and first order accuracy for $\left[\frac{\partial p^{2}}{\partial x_{1}^{2}}\right]$. We also obtain the desired accuracy for the other Cartesian jump conditions (not shown here).

The number of CG iterations required in computing $\left[\frac{\partial p}{\partial x_{i}}\right]$ (Section 2.1) remains relatively constant when the triangular meshes are refined for an interface, as indicated by Table 1 . Note that as the meshes for a cone or a cylinder are refined, some of the triangular panels become very thin, but the relatively constant iteration numbers in the CG method suggests that the method is not sensitive to the distortion of the meshes. This is expected because the coefficient matrix of the linear system in Eqn. (8) depends on the connectivity but not the geometry of a mesh. Only drastic changes in mesh topology may change the condition number of the coefficient matrix.

Lastly, we use the computed Cartesian jump conditions to solve a Poisson equation subject to given principal jump conditions across a closed interface in $3 \mathrm{D}$. The mathematical description of the test problem is

$$
\begin{aligned}
& \Delta p=f \quad \text { in } \quad \Omega \\
& {[p]=J_{p}, \quad\left[\frac{\partial p}{\partial \mathbf{n}}\right]=J_{n} \quad \text { and } \quad[\Delta p]=[f] \quad \text { on } \quad \Gamma,} \\
& \frac{\partial p}{\partial \mathbf{n}}=b \quad \text { on } \quad \partial \Omega,
\end{aligned}
$$




\begin{tabular}{cc|cc|cc}
\hline \hline \multicolumn{2}{c|}{ cube } & \multicolumn{2}{c|}{ cylinder } & cone & \\
\hline vertices/panels & iter & vertices/panels & iter & vertices/panels & iter \\
\hline $1352 / 2700$ & 23 & $5762 / 11520$ & 22 & $4322 / 8640$ & 22 \\
$5768 / 11532$ & 23 & $23810 / 47616$ & 22 & $17858 / 35712$ & 22 \\
$23816 / 47628$ & 23 & $96770 / 193536$ & 23 & $72578 / 145152$ & 22 \\
$96776 / 193548$ & 23 & $390146 / 780288$ & 24 & $292610 / 585216$ & 24 \\
$390152 / 780300$ & 23 & $1566722 / 3133440$ & 25 & $1175042 / 2350080$ & 25 \\
\hline \hline
\end{tabular}

Table 1: The number of CG iterations vs the number of vertices/panels for a cube, a cylinder and a cone.

where $\Omega$ is a rectangular domain with the boundary $\partial \Omega$, and $\Gamma$ is the interface in the domain. The right-hand side of the Poisson equation is

$$
f\left(x_{1}, x_{2}, x_{3}\right)= \begin{cases}-3 \sin \left(x_{1}\right) \sin \left(x_{2}\right) \sin \left(x_{3}\right), & \text { outside } \Gamma, \\ 3 \mathrm{e}^{-x_{1}} \mathrm{e}^{-x_{2}} \mathrm{e}^{-x_{3}}, & \text { inside } \Gamma .\end{cases}
$$

which is constructed using the analytical solution $p$ given in Eqn. (19).

With the computed Cartesian jump conditions, the Poisson problem can be discretized on a uniform Cartesian grid of the spatial step $h$ according to Eqn. (1), as described in Section 1. We use FFT from fftpack in serial computing (or MG from hypre in parallel computing) to solve the discretized Poisson problem and obtain the numerical solution. Fig. 4 shows the contours of the numerical solution at two slices of the computational domain for an interface of cone shape. As expected, we observe sharp jumps in the solution contours. Fig. 5 demonstrates that we achieve the desired second order accuracy of our solution in the infinity norm for an interface of sphere, cube, cylinder or cone shape. In the grid refinement study for Fig. 5, the Cartesian grid and the triangular mesh are refined together to keep the same order of sizes.

\section{Conclusions}

In this paper, we present and test a method to compute the necessary Cartesian jump conditions from the given principal jump conditions for a function that is discontinuous across an interface in 3D using triangular mesh representation of the interface. All the necessary Cartesian jump conditions are computed very efficiently with desired accuracy. 
(a)

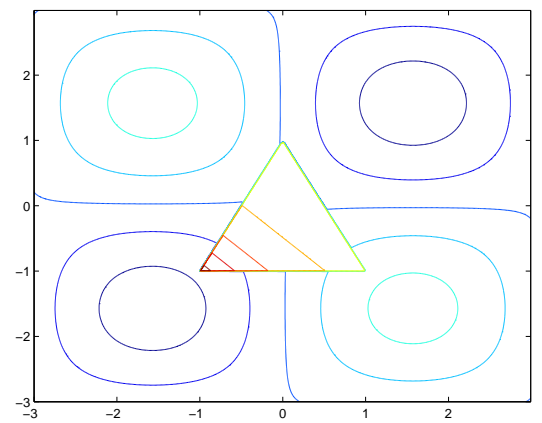

(b)

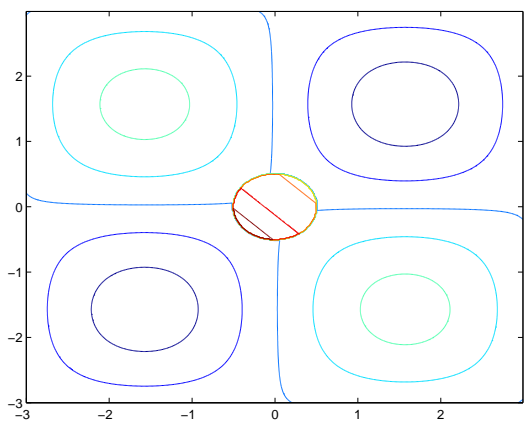

Figure 4: Contours of the solution at two slices for a Poisson equation subject to an interface with the shape of a cone: (a) an $x_{1} x_{3}$ slice, (b) an $x_{1} x_{2}$ slice.

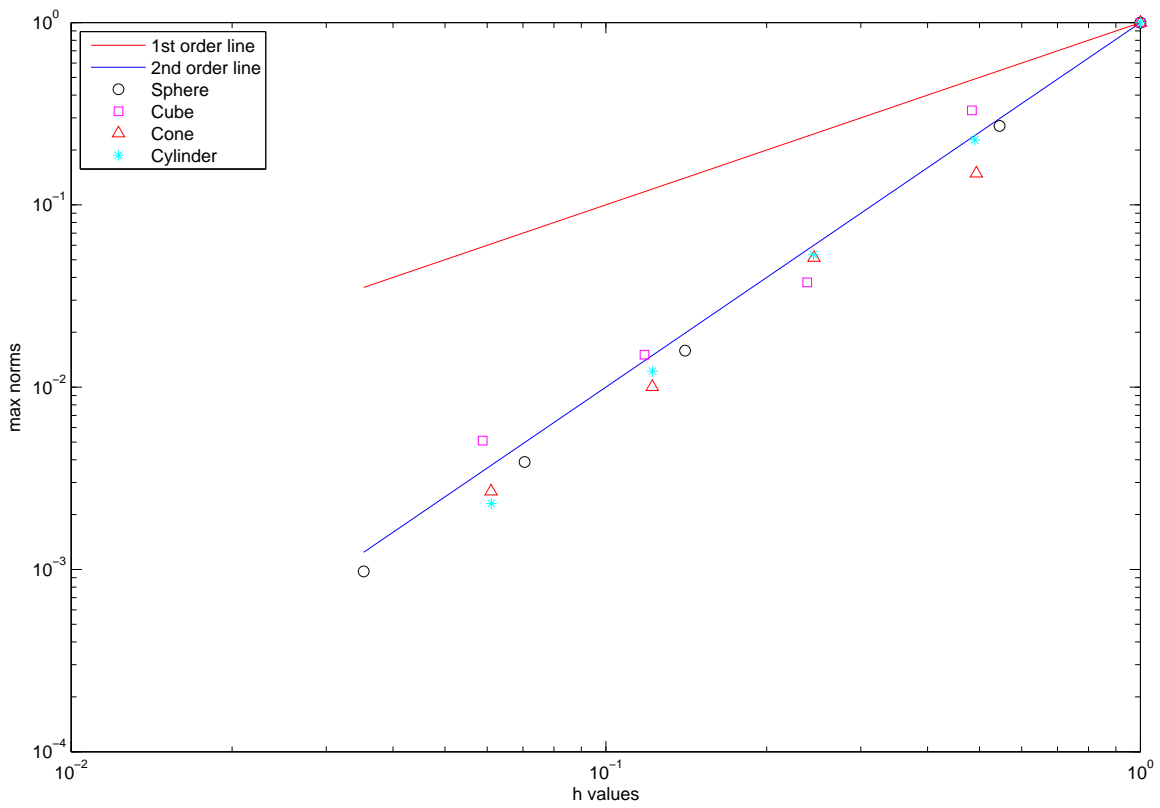

Figure 5: The scaled infinity norms of the errors versus the scaled sizes of triangular meshes and Cartesian grids for the computed solution $p$ of a Poisson equation. 
We first establish a small $3 \times 3$ linear system for the Cartesian jump conditions of the first-order derivatives of the function at the middle point (the point $M$ in Fig. 2 (a)) inside each triangular panel of the triangular mesh by differentiating the jump condition of the function itself on the panel. The Cartesian jump conditions of the first-order derivatives at the vertices of the mesh are related through interpolation with those at the middle points as a SPD system. The SPD system is solved using CG, which needs only a small number of iterations regardless of the interface shape, the mesh size and the mesh distortion. By differentiating the Cartesian jump conditions of the first-order derivatives along the edges of the mesh, we then establish a small over-determined $7 \times 6$ linear system for the Cartesian jump conditions of the second-order derivatives at at each vertex. This linear system has a unique solution and can be solved analytically. We also present how to interpolate from the vertices the necessary Cartesian jump conditions at the intersections of the interface and Cartesian grid lines. The jump corrections for finite difference stencils along the grid lines can then be obtained. Finally, we test our method by using the computed Cartesian jump conditions in the IIM to solve a Poisson equation subject to an interface with the shape of a sphere, cube, cylinder or cone. The results demonstrate the expected secondorder accuracy of the solution in the infinity norm.

The triangular mesh representation is simple and robust to represent a complex or non-smooth interface. Our contributions in this paper therefore enhance the robustness of the IMM to handle complex or non-smooth interfaces in applications, including fluid-solid interaction.

\section{Acknowledgment}

This work is supported by the NSF grant DMS-1320317.

\section{References}

[1] Huaxiong Huang and Zhilin Li, Convergence analysis of the immersed interface method, IMA J. Numer. Anal., 19 (4), pp. 583-608 (1999)

[2] Randall LeVeque and Zhilin Li, The immersed interface method for elliptic equations with discontinuous coefficients and singular forces, SIAM J. Numer. Anal., 31, pp. 1019-1044 (1994) 
[3] Zhilin Li and Kazufumi Ito, The Immersed Interface Method - Numerical Solutions of PDEs Involving Interfaces and Irregular Domains, SIAM Frontiers in Applied mathematics, 33, ISBN: 0-89971-609-8 (2006)

[4] Mark N. Linnick and Hermann F. Fasel, A high-order immersed interface method for simulating unsteady incompressible flows on irregular domains, J. Comput. Phys., 204, pp. 157-192 (2005)

[5] Andreas Wiegmann and Kenneth P. Bube, The Explicit-Jump Immersed Interface Method: Finite Difference Methods for PDES with Piecewise Smooth Solutions, SIAM J. Numer. Ana., Vol. 37, No. 3, pp. 827-862 (2000)

[6] Sheng Xu and Z. Jane Wang, Systematic Derivation of Jump Conditions for the Immersed Interface Method in Three-Dimensional Flow Simulation, SIAM J. Sci. Comput., Vol. 27, No. 6, pp. 1948-1980 (2006)

[7] Sheng Xu and Z. Jane Wang, A 3D immersed interface method for fluid-solid interaction, Comput. Methods Appl. Mech. Engrg., vol. 197, pp. 2068-2086 (2008)

[8] Sheng $\mathrm{Xu}$, The immersed interface method for simulating prescribed motion of rigid objects in an incompressible viscous flow, J. Comput. Phys., 227, pp. 5045-5071 (2008)

[9] Sheng Xu, Singular Forces in the Immersed Interface Method for Rigid Objects in 3D, Appl. Math. Lett., 22, pp.827-833 (2009)

[10] Sheng Xu, A boundary condition capturing immersed interface method for 3D rigid objects in a flow, J. Comput. Phys., 230, pp. 7176-7190 (2011) 